\title{
LOCAL FATOU THEOREM AND AREA THEOREM FOR SYMMETRIC SPACES OF RANK ONE
}

\author{
BY
}

\author{
A. KORÁNYI AND R. B. PUTZ( $\left.{ }^{1}\right)$
}

ABSTRACT. The classical results for the unit disc mentioned in the title are extended to harmonic functions on symmetric spaces of rank one.

Introduction. The study of the local boundary behavior of harmonic functions has a long history. For the unit disc, Privalov obtained a local version of Fatou's Theorem, and Marcinkiewicz and Zygmund, and Spencer proved the area theorem (see Chapter 14 of Zygmund [9]). These results were generalized to Euclidean half spaces by Calderón [1] and Stein [7]. The local Fatou Theorem for symmetric spaces of rank one is stated in [4] and [5] but the proof sketched there contains an error (the function $r_{n}$ on p. 514 of [4] is not always harmonic). For the basic idea of the present approach, proving a generalized area theorem by essentially Stein's method and deriving the local Fatou Theorem from it, we are indebted to C. Fefferman. For the special case of Hermitian hyperbolic space such a proof has already been carried out in [6]. The method used in the present paper, which makes more systematic use of the intrinsic geometry of the space, both simplifies the latter proof and generalizes the result to all rank one symmetric spaces. For holomorphic functions in strictly pseudo-convex domains with $C^{2}$ boundaries analogous theorems are also known [8]. For spaces of higher rank it is not clear if a similar result holds, since even the simplest case of the polydisc presents serious new difficulties.

In $\S 1$ we recall some known facts about rank one symmetric spaces and state the theorem. In $\S 2$ we prove some lemmas which incorporate most of the technical details of the proof of the main result, which is given in $\S 3$.

1. Throughout, $X$ will be a symmetric space of noncompact type and of rank one, $G$ will be its connected group of isometries, $K$ the isotropy group at a fixed point of $X$ that will be denoted $0, \boldsymbol{g}=\mathfrak{l}+\mathfrak{p}=\mathfrak{l}+\mathfrak{a}+\mathfrak{n}$ will be the usual Cartan and Iwasawa decompositions of the Lie algebra $g$ of $G$, and $\bar{n}$ the image

Received by the editors July $11,1975$.

AMS (MOS) subject classifications (1970). Primary 22E30; Secondary 43A85.

Key words and phrases. Symmetric spaces, boundary behavior of harmonic functions.

(1) Both authors were partially supported by the National Science Foundation under Grant GP-28448. 
of $\mathfrak{n}$ under the Cartan involution. The restricted roots are $\pm \lambda$ and possibly $\pm 1 / 2 \lambda$; $2 \rho$ is the sum of the positive roots with multiplicities counted. We choose $H \in$ a such that $\lambda(H)=2$ and write $m=2 \rho(H)$. The positive multiples of $H$ now form the positive Weyl chamber. Write $\bar{N}, A$ for the analytic subgroups of $G$ corresponding to $\overline{\mathfrak{n}}, \mathrm{a} . \bar{N} A$ is a group since $A$ normalizes $\bar{N}$, and every element of $X$ can be uniquely written in the form $n a \cdot O$ or $n \exp t H \cdot O$ with $n \in \bar{N}, a \in A$, $t \in \mathbf{R}$.

$X$ has a $G$-invariant Riemannian metric; we denote the gradient and the Laplacian by $\nabla$ and $\Delta$ respectively, the length of a tangent vector $v$ by $\|v\|$, and the invariant volume element by $d \mu$. The function $\varphi$ defined by $\varphi(n \exp t H \cdot o)$ $=e^{-m t}$ is harmonic (i.e. $\Delta \varphi=0$ ) and any power of it is an eigenfunction of the Laplacian.

On $\bar{N}$ we fix a Haar measure and a smooth homogeneous gauge, i.e. a positive real-valued $C^{\infty}$-function || on $\bar{N}-\{e\}$ such that $\left|n^{\exp t H}\right|=e^{-t}|n|$ and $\left|n^{-1}\right|=|n|$. It is obvious that such a gauge exists, and that any two homogeneous gauges are equivalent, in the sense that their ratios are bounded on $\bar{N}-$ $\{e\}$. We have $\left|n n^{\prime}\right| \leqslant k\left(|n|+\left|n^{\prime}\right|\right)$, and, denoting $b(r)=\{n \in \bar{N}:|n|<r\}$ we have meas $(b(r))=c r^{m}$ with some universal constants $k$ and $c$. This is proved in [5] for a particular gauge that is not smooth, and it clearly remains true for any homogeneous gauge.

On the Furstenberg boundary $G / M A N$ of $X, \bar{N}$ has an open dense orbit on which $\bar{N}$ is simply transitive. For simplicity, identifying $\bar{N}$ with its orbit, we talk about the "boundary point $n$ ", $(n \in \bar{N})$. Similarly to [5] we define the "admissible regions" $\Gamma_{\alpha}^{\top}(n)$ for $\alpha>0, \tau \in R$ by

$$
\begin{aligned}
\Gamma_{\alpha}^{\tau}(n) & =\left\{n(\exp t H) n^{\prime} \cdot 0:\left|n^{\prime}\right|<\alpha, t>\tau\right\} \\
& =\left\{n n^{\prime}(\exp t H) \cdot 0:\left|n^{\prime}\right|<\alpha e^{-t}, t>\tau\right\} .
\end{aligned}
$$

We say that a function $f$ defined on $X$ is admissibly convergent at $n \in \bar{N}$ if, for every $\alpha>0$ and some (hence every) $\tau, \lim f\left(n_{1} \exp t H \cdot 0\right)$ exists as $t \rightarrow \infty$ and $n_{1} \exp t H \cdot o \in \Gamma_{\alpha}^{\tau}(n)$. These definitions are equivalent to those in [5] because of the equivalence of all homogeneous gauges. In fact, the whole statement of the theorem is indifferent to the particular gauge used on $\bar{N}$.

THEOREM. Let $E$ be a measurable subset of $\bar{N}$ and $f$ a harmonic function on $X$. Then the following are equivalent:

(i) For almost every $n \in E$, there exists $\alpha>0, \tau \in \mathbf{R}$ such that $f$ is bounded in $\Gamma_{\alpha}^{\tau}(n)$.

(ii) $f$ is admissibly convergent at almost every $n \in E$.

(iii) For almost every $n \in E$, there exists $\alpha>0, \tau \in \mathbf{R}$ such that 


$$
\int_{\Gamma_{\alpha}^{\tau}(n)}\|\nabla f\|^{2} d \mu<\infty .
$$

(iii') For every fixed $\alpha>0$ and $\tau \in \mathbf{R}, \int_{\Gamma \downarrow}(n)\|\nabla f\|^{2} d \mu<\infty$ for almost every $n \in E$.

The proof is divided into three parts: (i) $\Rightarrow$ (iii'), (iii) $\Rightarrow$ (i), and (i) and (iii') $\Rightarrow$ (ii). Since (ii) $\Rightarrow$ (i) and (iii') $\Rightarrow$ (iii) are trivial, this is enough. It will be clear from the proof that it is not even necessary to have $f$ everywhere defined; the theorem holds with $f$ defined only in some $\Gamma_{\alpha}^{\tau}(n)$ at each $n \in E$. We also note that the equivalence of (iii) and (iii') is an immediate consequence of Lemmas 2,3 , and 4 . Finally we note that since the result is local, we may assume that $E$ is bounded.

2. The following lemma is an immediate consequence of the fact that almost every point of $E$ is a point of density with respect to the sets $n b(r)$.

LEMmA 1. Let $E$ be a bounded measurable subset of $\bar{N}$ and $\epsilon>0$. Then there exists a constant, $r_{0}>0$, and a closed subset $D$ of $E$ such that meas $(E-D)<$ $\epsilon$, and for $n_{0} \in D$ and $r \leqslant r_{0}$, meas $\left(E \cap n_{0} b(r)\right) \geqslant(1-\epsilon) \operatorname{meas}(b(r))$.

The next three lemmas will be used to replace condition (iii) with an integration over unions of admissible regions.

DEFINITION. If $E$ is a bounded measurable subset of $\bar{N}$,

$$
W_{\alpha}^{\tau}(E)=\bigcup_{n \in E} \Gamma_{\alpha}^{\tau}(n)
$$

LEMMA 2. If $n_{0}$ is a point of density of $E$ with respect to the family of sets $n b(r)$; then for $\alpha>0, \alpha_{0}>0, \tau \in R$, there exists $\tau_{0}$ such that $\Gamma_{\alpha_{0}}^{\tau}\left(n_{0}\right) \subset$ $W_{\alpha}^{\tau}(E)$.

Proof. This is Lemma 5 of [4] and the proof is essentially that of Calderón.

LEMMA 3. If $f$ is a nonnegative locally integrable function on $W_{\alpha}^{\tau}(E)$ and $\int_{W \tau_{\alpha}(E)} f(n \exp t H \cdot o) e^{-m t} d \mu<\infty$, then $\int_{\Gamma_{\alpha^{\prime}}^{\tau}\left(n^{\prime}\right)} f(n \exp t H \cdot o) d \mu<\infty$ for all $\alpha^{\prime}>0$ and almost every $n^{\prime} \in E$.

Proof. It is sufficient to prove that $\int_{E} d n^{\prime} \int_{\Gamma_{\alpha}^{\prime},\left(n^{\prime}\right)} f(n \exp t H \cdot o) d \mu<$ $\infty$. By Lemma 2 we may assume that $\alpha^{\prime}=\alpha$. With $\chi$ the characteristic function of $\Gamma_{\alpha}^{\tau}\left(n^{\prime}\right)$ we have:

$$
\begin{aligned}
\int_{E} d n^{\prime} \int_{\Gamma_{\alpha}^{\tau}\left(n^{\prime}\right)} f(n \exp t H \cdot o) d \mu & =\int_{E} d n^{\prime} \int_{W_{\alpha}^{\tau}(E)} \chi(n \exp t H \cdot o) f(n \exp t H \cdot o) d \mu \\
& =\int_{W_{\alpha}^{\tau}(E)} d \mu f(n \exp t H \cdot o) \int_{E} \chi(n \exp t H \cdot o) d n^{\prime} .
\end{aligned}
$$


Now

$$
\int_{E} \chi(n \exp t H \cdot 0) d n^{\prime} \leqslant \int_{n b\left(e^{-t} \alpha\right)} d n^{\prime}=c \alpha^{m} e^{-m t}
$$

and the assertion follows.

In light of Lemma 2, and the observation that we are always dealing with locally bounded, locally integrable functions, we may assume that the truncation level, $\tau$, is fixed, say $\tau=0$, and henceforth we suppress the superscript.

LEMMA 4. Suppose $f$ is nonnegative and locally integrable in a neighborhood of $W_{\alpha}(E)$, and for each $n^{\prime} \in E$ there exists $\alpha\left(n^{\prime}\right)>0$ such that $\int_{\Gamma_{\alpha\left(n^{\prime}\right)}\left(n^{\prime}\right)} f(n \exp t H \cdot 0) d \mu<\infty$. Then for every $\epsilon>0, \alpha>0$ there exists a closed set $D \subset E$ such that meas $(E-D)<\epsilon$ and $\int_{W_{\alpha}(D)} f(n \exp t H \cdot o) e^{-m t} d \mu$ $<\infty$.

Proof. Let $E_{j, k}=\left\{n^{\prime} \in E: \int_{\Gamma_{1 / j}\left(n^{\prime}\right)} f(n \exp t H \cdot o) d \mu \leqslant k\right\}$. Then $E_{j, k}$ $\subset E_{j+1, k} \subset E_{j+1, k+1}$ and $\bigcup_{j, k} E_{j, k}=E$. Thus we can choose $j_{0}, k_{0}, E_{0}=$ $E_{j_{0}, k_{0}}, \alpha^{\prime}=1 / j_{0}$ such that meas $\left(E-E_{0}\right)<\epsilon / 2$ and $\int_{\Gamma_{\alpha^{\prime}}\left(n^{\prime}\right)} f(n \exp t H \cdot 0) d \mu$ $\leqslant k_{0}$ for all $n^{\prime} \in E_{0}$. By Lemma 1 there exists $t_{0}$ and $D \subset E_{0}$ such that meas $\left(E_{0}-D\right)<\epsilon / 2$ and, for $n^{\prime} \in D$ and $t \geqslant t_{0}$,

$$
\operatorname{meas}\left(E_{0} \cap n^{\prime} b\left(\alpha e^{-t}\right)\right) \geqslant(1-\epsilon / 2) \operatorname{meas}\left(b\left(\alpha e^{-t}\right)\right) .
$$

By Lemma 2 we may assume $\alpha=\alpha^{\prime}$. Thus, for $n^{\prime} \in D, t \geqslant t_{0}$, and $\chi$ as in the proof of Lemma 3,

$$
\int_{E_{0}} \chi(n \exp t H \cdot o) d n=\operatorname{meas}\left(E_{0} \cap n^{\prime} b\left(\alpha e^{-t}\right)\right) \geqslant(1-\epsilon / 2) c \alpha^{m} e^{-m t}
$$

Thus:

$$
\begin{aligned}
k_{0} \operatorname{meas}\left(E_{0}\right) & \geqslant \int_{E_{0}} d n^{\prime} \int_{\Gamma_{\alpha}\left(n^{\prime}\right)} f(n \exp t H \cdot o) d \mu \\
& =\int_{E_{0}} d n^{\prime} \int_{W_{\alpha}\left(E_{0}\right)} \chi(n \exp t H \cdot o) f(n \exp t H \cdot o) d \mu \\
& =\int_{W_{\alpha}\left(E_{0}\right)} d \mu f(n \exp t H \cdot o) \int_{E_{0}} \chi(n \exp t H \cdot 0) d n^{\prime} \\
& \geqslant\left(1-\frac{\epsilon}{2}\right) c \alpha^{m} \int_{W_{\alpha}(D)} f(n \exp t H \cdot o) e^{-m t} d \mu .
\end{aligned}
$$

The next step is to replace the regions $W_{\alpha}(E)$ by regions on which we can apply Green's Theorem. Let $\left\{n_{j}\right\}_{1}^{\infty}$ be a countable dense sequence in $E$, and

$$
W_{k}=\bigcup_{1<j<k}\left(\Gamma_{\alpha}\left(n_{j}\right) \cap\{n \exp t H \cdot 0: t<k\}\right) .
$$


Then $W_{k}$ is a bounded region with piecewise smooth boundary, $W_{k} \subset W_{k+1}$, and $\bigcup_{k} W_{k}=W_{\alpha}(E)$. We will be using estimates on the surface element of $\partial W_{k}$, the boundary of $W_{k}$, and we will need to consider separately:

$$
\begin{aligned}
& S_{k, 0}=\partial W_{k} \cap\{n \exp t H \cdot o: t=0\}, \text { the top; } \\
& S_{k, 1}=\partial W_{k} \cap\{n \exp t H \cdot 0: 0<t<k\}, \text { the side; } \\
& S_{k, 2}=\partial W_{k} \cap\{n \exp t H \cdot o: t=k\}, \text { the bottom. }
\end{aligned}
$$

LEMma 5. Let ds be the surface element on $S_{k, 1}$ or $S_{k, 2}, \partial / \partial n$ the outward pointing unit normal to $S_{k, 1}$ or $S_{k, 2}$, and $d n$ the left invariant volume form of $\bar{N}$. Then there exist constants $C_{1}, C_{2}, C_{3}>0$, independent of $k$, such that

(a) $-C_{1}(\partial / \partial n) e^{-m t} \leqslant e^{-m t} \leqslant-C_{2}(\partial / \partial n) e^{-m t}$,

(b) $-(\partial / \partial n) e^{-m t} d s=C_{3} \pi^{*}(d n)$, where $\pi: S_{k, 1} \cup S_{k, 2} \rightarrow \bar{N}$ by $\pi(n \exp t H \cdot o)=n$.

Proof. Let $X_{1}, \ldots, X_{r}$ be a basis of $\overline{\mathfrak{n}}$. Then

$$
\exp \sum x_{i} X_{i} \cdot \exp t H \cdot o \rightarrow\left(x_{1}, \ldots, x_{r}, t\right)
$$

is a global coordinate system on $X . S_{k, 1} \cup S_{k, 2}$ is a piecewise smooth manifold; $\pi$ is injective, and $\pi$ followed by the canonical coordinates of $\bar{N}$ is a global coordinate system on $S_{k, 1} \cup S_{k, 2}$, smooth on each smooth piece.

Step 1. The coordinate vector field $\partial / \partial t$ has constant length and is perpendicular to $\partial / \partial x_{i}, 1 \leqslant i \leqslant r$, on $X$. In fact, $\partial / \partial t$ is immediately seen to be $\bar{N} A-\cdot$ invariant and it is well known [3] that $\partial /\left.\partial t\right|_{0}$ is perpendicular to the orbit $\bar{N} \cdot 0$. Since $\bar{N} A$ is a transitive group of isometries, $\partial / \partial t$ has constant length. Since the element $n a \in \bar{N} A$ maps $\bar{N} \cdot o$ onto $\overline{N a} \cdot o, \partial / \partial t$ is everywhere perpendicular to $\bar{N}$-orbits, and hence to $\partial / \partial x_{i}, 1 \leqslant i \leqslant r$.

Step 2. $\nabla e^{-m t}=-C e^{-m t} \partial / \partial t . \nabla e^{-m t}$ is normal to the surfaces $e^{-m t}=$ constant, i.e. to the $\bar{N}$-orbits, and therefore, by Step 1 , is proportional to $\partial / \partial t$. To find the proportionality factor we recall that $e^{-m t}$ is harmonic and $e^{-2 m t}$ is an eigenfunction of $\Delta$. Thus $\left\|\nabla e^{-m t}\right\|^{2}=1 / 2 \Delta e^{-2 m t}=C e^{-2 m t}$.

Step 3. $(\partial / \partial n \mid \partial / \partial t) \geqslant C^{\prime}>0$ everywhere on $S_{k, 1} \cup S_{k, 2}$. For $S_{k, 2}$ this is clear, since, by Step 2, $\partial / \partial n=C \partial / \partial t$ for some $C>0 . S_{k, 1}$ is a finite union of parts of the boundaries of $\Gamma_{\alpha}\left(n_{j}\right), 1 \leqslant j \leqslant k$. We may assume that $n_{j}=e$, since $n_{j}$ is an isometry and $\left(n_{j}\right)^{*} \partial / \partial t=\partial / \partial t$. We may also assume that we are at a point $x=n \cdot o$, since we can reach such a point by applying some $a \in A ; a$ is an isometry which preserves $\Gamma_{\alpha}(e)$, hence $a_{*} \partial / \partial n=\partial / \partial n$ and $a_{*} \partial / \partial t=\partial / \partial t$. Now $\partial \Gamma_{\alpha}(e)=\left\{n \exp t H \cdot o: e^{t}|n|=\alpha\right\}$, and thus, for some proportionality factor $\varphi(x)$, by Step 2 , 


$$
\begin{aligned}
\partial /\left.\partial n\right|_{x} & =\left.\varphi(x) \nabla\right|_{x}\left(e^{t}|n|\right)=\left.\varphi(x)\left(|n| \nabla e^{t}+e^{t} \nabla|n|\right)\right|_{x} \\
& =\varphi(x)\left(C \frac{\partial}{\partial t}+\nabla|n|\right) .
\end{aligned}
$$

Now $\nabla|n|$ is bounded on the compact set $|n|=\alpha$, and, by Step 1 , is perpendicular to $\partial / \partial t$. Thus $\varphi(x)$ is bounded away from zero, and we have Step 3.

The second inequality in (a) follows from Steps 2 and 3 since:

$$
-\frac{\partial}{\partial n} e^{-m t}=-\left(\frac{\partial}{\partial n} \mid \nabla e^{-m t}\right)=C\left(\frac{\partial}{\partial n} \mid e^{-m t} \frac{\partial}{\partial t}\right) \geqslant C C^{\prime} e^{-m t} .
$$

The first half of (a) is trivial since $(\partial / \partial n \mid \partial / \partial t)$ is bounded.

To prove (b), choose $n_{0} a \cdot o \in S_{k, 1} \cup S_{k, 2}$ and let $\delta>0, C=$ \{na $o$ : $\left.n \in n_{0} b(\delta)\right\}$. By Green's Theorem

$$
0=\int_{w_{k} \cap C} \Delta e^{-m t} d \mu=\int_{\partial\left(w_{k} \cap C\right)} \frac{\partial}{\partial n} e^{-m t} d s .
$$

$\partial\left(W_{k} \cap C\right)$ consists of parts of $\partial W_{k}$ and a part of $\partial C$. By Step $1, \partial e^{-m t} / \partial n$ vanishes on the latter and therefore we have:

$$
\int_{\left(s_{k, 1} \cup s_{k, 2}\right) \cap C} \frac{\partial}{\partial n} e^{-m t} d s=-\int_{\left\{n \cdot 0: n \in n_{0} b(\delta)\right\}} \frac{\partial}{\partial n} e^{-m t} d s .
$$

In the latter integral $d s$ is a constant multiple of $\pi^{*}(d n)$ since both are $\bar{N}$-invariant forms of maximal degree, and $\partial e^{-m t} / \partial n$ is a constant. Hence we have (b).

The next two propositions give estimates on the gradient of a harmonic function, which we will use in conjunction with the bounds given by (iii) and (i) respectively. They are valid for $X$ of arbitrary rank.

PROPOSITON 6. If $f$ is harmonic, $x \in X$, and $B_{r}(x)$ is the ball of radius $r$ about $x$, then there exists a constant $C$, depending only on $r$, such that $\|\nabla f(x)\|^{2}$ $\leqslant C \int_{B_{r}(x)}\|\nabla f(z)\|^{2} d \mu$.

Proof. Choose $g \in G$ such that $g \cdot o=x$. Then $\|\nabla f(x)\|=\|\nabla f(g \cdot 0)\|$ $=\|\nabla(f \circ g)(0)\|$. Thus it is sufficient to prove the result for $x=0$. Take an orthonormal basis of $p$ and let $\left\{X_{1}, \ldots, X_{n}\right\}$ be the induced Killing vector fields on $X$. Then $X_{i} f$ is harmonic $(1 \leqslant i \leqslant n)$ because of the $G$-invariance of $\Delta$. With $g_{i j}(z)=\left.g\left(X_{i}, X_{j}\right)\right|_{z},\left[g^{i j}(z)\right]=\left[g_{i j}(z)\right]^{-1}$, we have $\|\nabla f(z)\|^{2}=$ $\Sigma_{i, j} g^{i j}(z)\left(X_{i} f\right)(z)\left(X_{j} f\right)(z)$ and $g^{i j}(0)=\delta(i, j)$. Now, using the mean value theorem, Schwarz's inequality, and noting that the smallest eigenvalue of $\left[g^{i j}(z)\right]$ has a positive minimum on $B_{r}(o)$, we have: 


$$
\begin{aligned}
\|\nabla f(o)\|^{2} & =\sum_{i}\left|X_{i} f(o)\right|^{2} \leqslant \frac{1}{\mu\left(B_{r}(o)\right)} \int_{B_{r}(o)} \sum_{i}\left|X_{i} f(z)\right|^{2} d \mu \\
& \leqslant C \int_{B_{r}(o)} \sum_{i, j} g^{i j}(z)\left(X_{i} f\right)(z)\left(X_{j} f\right)(z) d \mu=C \int_{B_{r}(o)}\|\nabla f\|^{2} d \mu .
\end{aligned}
$$

CoRollaRY 7. If $\int_{\Gamma_{\alpha}(n)}\|\nabla f\|^{2} d \mu<\infty$, then, for $\alpha^{\prime}<\alpha,\left\|\nabla f\left(n^{\prime} \exp t H \cdot o\right)\right\|$ $=o(1)$ as $t \rightarrow \infty$ for $n^{\prime} \exp t H \cdot o \in \Gamma_{\alpha^{\prime}}(n)$.

Proof. If $\alpha^{\prime}<\alpha$, there exists an $r>0$ such that $g \cdot o \in \Gamma_{\alpha^{\prime}}(n)$ implies $g \cdot B_{r}(o) \subset \Gamma_{\alpha}(n)$, and the result is immediate from the proposition above.

Proposition 8. If $f$ is harmonic and bounded in $\Gamma_{\alpha}(n)$, and $\alpha^{\prime}<\alpha$, then $\|\nabla f\|$ is bounded in $\Gamma_{\alpha^{\prime}}(n)$.

Proof. Choose $r>0$ such that $g \cdot o \in \Gamma_{\alpha^{\prime}}(n)$ implies $g \cdot B_{r}(o) \subset \Gamma_{\alpha}(n)$. There exists a kernel $K_{r}$ such that, for $h$ harmonic on $\overline{B_{r}(o)}$ and $z \in B_{r}(o)$,

and thus

$$
h(z)=\int_{\partial B_{r}(o)} K_{r}(z, x) h(x) d s,
$$

$$
\nabla h(z)=\int_{\partial B_{r}(o)} \nabla_{z} K_{r}(z, x) h(x) d s .
$$

If $g \cdot o \in \Gamma_{\alpha^{\prime}}(n)$, then $f \circ g$ is harmonic on $\overline{B_{r}(o)}$ and we have

$$
\|\nabla(f \circ g)(z)\|^{2} \leqslant c \int_{\partial B_{r}(o)}\left\|\nabla_{z} K_{r}(z, x)\right\|^{2}|(f \circ g)(x)|^{2} d s .
$$

For $z=o$ and $x \in \partial B_{r}(o),\left\|\nabla_{z} K_{r}(z, x)\right\|^{2}$ is bounded by a constant depending only on $r$ and we have

$$
\|\nabla(f \circ g)(o)\|^{2} \leqslant c \sup _{x \in \partial B_{r}(o)}|(f \circ g)(x)|^{2} .
$$

We will be using Poisson integrals, and we now recall some of their properties [5, p. 398]. If $g$ is a function on $\bar{N}$, then its Poisson integral is $G(n \exp t H \cdot o)=\left(g * P_{t}\right)(n)$; the kernel is positive, integrable on $\bar{N}$ and has the homogeneity: $P_{t+t_{1}}\left(n^{\exp t_{1} H}\right)=P_{t}(n) e^{m t_{1}}$.

LEMma 9. For every $\epsilon>0$ and $M>0$ there exists $R>0$ such that if $g$ $\in L^{\infty}(\bar{N})$ with $\|g\|_{\infty} \leqslant M$ and $g\left(n_{1}\right)>s+\epsilon$ for all $n_{1} \in n b\left(R e^{-t}\right)$ with some $n$ $\in \bar{N}, t \in \mathrm{R}, s \in \mathrm{R}$, then $\left(g * P_{t}\right)(n)>s$.

Proof. It is sufficient to assume that $n=e$. Then

$$
\begin{aligned}
\left(g * P_{t}\right)(e)= & \int_{\bar{N}} g\left(n_{1}\right) P_{t}\left(n_{1}^{-1}\right) d n_{1}=\int_{n_{1} \in b\left(R e^{-t}\right)} g\left(n_{1}\right) P_{t}\left(n_{1}^{-1}\right) d n_{1} \\
& +\int_{n_{1} \notin b\left(R e^{-t}\right)} g\left(n_{1}\right) P_{t}\left(n_{1}^{-1}\right) d n_{1} .
\end{aligned}
$$


Now, using the homogeneity of $P$, for $R$ sufficiently large,

$$
\int_{n_{1} \notin b\left(R e^{-t}\right)} g\left(n_{1}\right) P_{t}\left(n_{1}^{-1}\right) d n_{1} \leqslant\|g\|_{\infty} \int_{n_{1} \notin b(R)} P_{0}\left(n_{1}^{-1}\right) d n_{1}<\frac{\epsilon}{2}
$$

and the result follows.

3. Proof of the Theorem.

(i) $\Rightarrow$ (iii'). We begin by imposing some uniform conditions. Let

$$
E_{j, k}=\left\{n \in E:|f| \leqslant k \text { in } \Gamma_{1 / j}(n)\right\} .
$$

Then $E_{j, k} \subset E_{j, k+1} \subset E_{j+1, k+1}$ and $\bigcup_{j, k} E_{j, k}=E$ since $f$ is admissibly bounded on $E$. Thus, for $\epsilon>0$, there exists $D^{\prime} \subset E$ and constants $M_{0}>0, \alpha_{0}>0$ such that meas $\left(E-D^{\prime}\right)<\epsilon / 2$, and $|f| \leqslant M_{0}$ on $W_{\alpha_{0}}\left(D^{\prime}\right)$. Now, given any $\alpha>0$, a similar argument based on Lemma 2 shows the existence of $D \subset D^{\prime}, \operatorname{meas}\left(D^{\prime}-D\right)$ $<\epsilon / 2$, such that $|f| \leqslant M$, with some $M$, on $W_{\alpha}(D)$. By Lemma 3 , it is sufficient to show that $\int_{W_{\alpha}(D)}\|\nabla f\|^{2} e^{-m t} d \mu<\infty$; and this is equivalent to showing that $\int w_{k}\|\nabla f\|^{2} e^{-m t} d \mu$ is uniformly bounded in $k$. Since $f$ and $e^{--m t}$ are harmonic, we have, by Green's Theorem:

$$
\begin{aligned}
\int_{W_{k}}\|\nabla f\|^{2} e^{-m t} d \mu & =\frac{1}{2} \int_{W_{k}}\left\{e^{-m t} \Delta f^{2}-f^{2} \Delta e^{-m t}\right\} d \mu \\
& =\frac{1}{2} \int_{\partial W_{k}}\left\{e^{-m t} \frac{\partial}{\partial n} f^{2}-f^{2} \frac{\partial}{\partial n} e^{-m t}\right\} d s \\
& \leqslant \int_{\partial W_{k}}\left\{e^{-m t}|f|\left|\frac{\partial f}{\partial n}\right|+\frac{1}{2}|f|^{2}\left|\frac{\partial}{\partial n} e^{-m t}\right|\right\} d s .
\end{aligned}
$$

But $|f| \leqslant M$, and, by Lemma 2 and Proposition $8,|\partial f / \partial n| \leqslant M^{\prime}$. Thus we have:

$$
\int_{W_{k}}\|\nabla f\|^{2} e^{-m t} d \mu \leqslant M M^{\prime} \int_{\partial W_{k}} e^{-m t} d s+\frac{M}{2} \int_{\partial w_{k}}\left|\frac{\partial}{\partial n} e^{-m t}\right| d s
$$

and the right-hand side is uniformly bounded by Lemma 5 .

(iii) $\Rightarrow$ (i). Let $\alpha>0, \epsilon>0$. By Lemmas 4 and 3 there exists $D_{0} \subset E$ such that

$$
\operatorname{meas}\left(E-D_{0}\right)<\frac{\epsilon}{2}, \quad \int_{W_{\alpha}\left(D_{0}\right)} e^{-m t}\|\nabla f\|^{2} d \mu=M<\infty,
$$

and (iii') holds for every point of $D_{0}$. By Corollary 7 there exists $D \subset D_{0}$ such that meas $\left(D_{0}-D\right)<\epsilon / 2$ and $\|\nabla f\| \leqslant M^{\prime}$ in $W_{2 \alpha}(D)$. Working with the set $W_{\alpha}(D)$ and defining $W_{k}$ as before, Green's Theorem gives:

$$
M \geqslant \frac{1}{2} \int_{\partial w_{k}}\left\{e^{-m t} \frac{\partial}{\partial n} f^{2}-f^{2} \frac{\partial}{\partial n} e^{-m t}\right\} d s .
$$


With $\partial W_{k}=S_{k, 0} \cup S_{k, 1} \cup S_{k, 2}$ as in Lemma 5, we rewrite this inequality as:

$$
\begin{aligned}
\frac{1}{2} \int_{s_{k, 1} \cup s_{k, 2}} f^{2}\left(-\frac{\partial}{\partial n} e^{-m t}\right) d s & \leqslant-\int_{s_{k, 1} \cup s_{k, 2}} e^{-m t} f \frac{\partial f}{\partial n} d s \\
& +\frac{1}{2} \int_{S_{k, 0}}\left\{f^{2} \frac{\partial}{\partial n} e^{-m t}-e^{-m t} \frac{\partial f^{2}}{\partial n}\right\} d s+M .
\end{aligned}
$$

$S_{k, 0}$ is contained in a bounded set on which each term of the integrand is bounded, and thus the integral over $S_{k, 0}$ is bounded independently of $k$. Also $|\partial f / \partial n| \leqslant\|\nabla f\|$ which is uniformly bounded by Corollary 7. (In the following $M$ denotes a generic constant, which may change with each appearance, but it is independent of $k$.) We therefore have:

$$
\int_{S_{k, 1} \cup s_{k, 2}} f^{2}\left(-\frac{\partial}{\partial n} e^{-m t}\right) d s \leqslant M \int_{S_{k, 1} \cup s_{k, 2}}|f| e^{-m t} d s+M .
$$

Applying Lemma 5 twice, and Schwarz's inequality in between, we have:

$$
\begin{aligned}
\int_{s_{k, 1} \cup s_{k, 2}} f^{2} e^{-m t} d s & \leqslant M \int_{S_{k, 1} \cup s_{k, 2}}|f| e^{-m t} d s+M \\
& \leqslant M\left\{\int_{S_{k, 1} \cup s_{k, 2}}|f|^{2} e^{-m t} d s\right\}^{1 / 2}+M
\end{aligned}
$$

and thus

$$
\int_{s_{k, 1} \cup s_{k, 2}} f^{2} e^{-m t} d s \leqslant M .
$$

We will now use this " $L^{2}$-boundedness" to prove admissible boundedness almost everywhere. Let

$$
f_{k}(n)= \begin{cases}|f(n \exp t H \cdot o)| & \text { if } n \exp t H \cdot o \in S_{k, 1} \cup S_{k, 2} \text { for some } t, \\ 0 & \text { otherwise, }\end{cases}
$$

and $F_{k}$ be the Poisson integral of $f_{k}$.

CLAIM. There exists a constant $M$, independent of $k$, such that $|f| \leqslant M+$ $M F_{k}$ on $W_{k}$.

Proof of ClaIM. By the maximum principle it is sufficient to prove the inequality on $\partial W_{k}$. Pick $r_{0}>0$. We can choose $M$ large so that $|f| \leqslant M$ on $S_{k, 0}$ and on $\left\{n \exp t H \cdot o \in S_{k, 1} \cup S_{k, 2}: t \leqslant r_{0}\right\}$. Choose $\delta>0, r>0(r<$ $r_{0}$ ) such that: if $n \exp t H \cdot o \in S_{k, 1} \cup S_{k, 2}$ and $t>r_{0}$ then, defining

$$
S=\left(S_{k, 1} \cup S_{k, 2}\right) \cap\left\{n_{1} \exp t_{1} H \cdot o: n_{1} \in n b\left(\delta e^{-t}\right)\right\}
$$


and

$$
C(\delta, r)=\left\{n_{1} \exp t_{1} H \cdot o: n_{1} \in n b\left(\delta e^{-t}\right),\left|t-t_{1}\right|<r\right\},
$$

we have $S \subset C(\delta, r) \subset W_{2 \alpha}(D)$. Recalling that $\|\nabla f\| \leqslant M^{\prime}$ in $W_{2 \alpha}(D)$ we have, for $n_{1} \exp t_{1} H \cdot o \in S$,

$$
\left|f\left(n_{1} \exp t_{1} H \cdot o\right)-f(n \exp t H \cdot o)\right| \leqslant C \sup \|\nabla f\| \leqslant M .
$$

By Lemma 5 , and in particular the proof of part (b), $\int_{\mathcal{S}} d s$ is bounded away from 0 . Thus

$$
\begin{aligned}
|f(n \exp t H \cdot o)| & \leqslant M+\left|f\left(n_{1} \exp t_{1} H \cdot o\right)\right| \\
& \leqslant M+M \int_{S}\left|f\left(n_{1} \exp t_{1} H \cdot o\right)\right| d s \\
& \leqslant M+M \int_{S}\left(f_{k} \circ \pi\right)\left(n_{1} \exp t_{1} H \cdot o\right) e^{m t_{1} \pi^{*}\left(d n_{1}\right)} \\
& \leqslant M+M e^{m t} \int_{\pi(S)} f_{k}\left(n_{1}\right) d n_{1} \cdot
\end{aligned}
$$

Now the homogeneity of the Poisson kemel shows that $P_{t}(n) \geqslant M e^{m t}$ for $|n|<$ $c e^{-t}$. Therefore (since $\left.\pi(S)=n b\left(\delta e^{-t}\right)\right)$,

$$
|f(x)| \leqslant M+M \int_{n b\left(c e^{-t}\right)} f_{k}\left(n_{1}\right) P_{t}\left(n_{1}^{-1} n\right) d n_{1} \leqslant M+M F_{k}(x)
$$

and we have established the claim. Now $\left\{f_{k}\right\}$ is a uniformly bounded sequence in $L^{2}(\bar{N})$ since, by Lemma 5 ,

$$
\int_{\bar{N}} f_{k}^{2} d n=\int_{s_{k, 1} \cup s_{k, 2}} f^{2} \pi^{*}(d n) \leqslant M \int_{s_{k, 1} \cup s_{k, 2}} f^{2} e^{-m t} d s \leqslant M .
$$

Thus there exists a subsequence that converges in $L^{2}(\bar{N})$ to, say, $f_{0}$. With $F_{0}$ the Poisson integral of $f_{0}$, the claim implies that $|f| \leqslant M+M F_{0}$ on $W_{\alpha}(D)$. Since Poisson integrals of $L^{2}$ functions converge admissibly almost everywhere [5] we know that $f$ is admissibly bounded almost everywhere on $D$.

(iii) and (i) $\Rightarrow$ (ii). Let $\epsilon>0$ be given.

Also let $\eta>0$; as in a previous argument we find $D_{1} \subset E$, meas $\left(E-D_{1}\right)$ $\left\langle\eta / 2\right.$ and $\alpha>0$ such that $f$ is bounded in $W_{\alpha}\left(D_{1}\right)$. By cutting up, if necessary, $E$ into finitely many sufficiently small pieces, we may assume that $W_{\alpha}\left(D_{1}\right)$ and $W_{\alpha}(D)$ below, are connected.

Now let $R$ be the number given by Lemma 9 corresponding to $\epsilon$ and to a bound $s$ of $|f|$ in $W_{\alpha}\left(D_{1}\right)$.

Note that if $x=n \exp k H \cdot o, x_{1}=n_{1} \exp k H \cdot o$ and $n_{1} \in n b\left(R e^{-k}\right)$, then the distance of these points is bounded (independently of $k$ ) by a multiple of $R$, and by Lemma 5 , they can be joined by a line $\gamma$ lying inside $\Gamma_{\alpha}(n) \cup$ $\Gamma_{\alpha}\left(n_{1}\right)$ such that if $n^{\prime} \exp t H \cdot o$ is on $\gamma$, then $t>k-c R$ and for the length of $\gamma$ we have $l(\gamma)<c^{\prime} R . \quad c$ and $c^{\prime}$ are independent of $k$. 
Now, using Corollary 7 and the usual argument we find $D \subset D_{1}$, meas $\left(D_{1}-D\right)$ $<\eta / 2$ and an integer $k_{0}$, such that $\|\nabla f\|<\epsilon / c^{\prime} R$ in $W_{\alpha}^{k} 0(D)$.

It follows that, if $k>k_{0}+c R$ and $x, x_{1}$ in $W_{\alpha}(D)$ are as above, then

$$
\left|f(x)-f\left(x_{1}\right)\right| \leqslant l(\gamma) \max _{\gamma}\|\nabla f\|<\epsilon .
$$

Now, for integers $k>k_{0}+c R$, we consider the new approximating regions

$$
W_{k}^{\prime}=\left\{n \exp t H \cdot o \in W_{\alpha}(D): t \leqslant k\right\} .
$$

We denote by $S_{k}$ the "bottom" of $\partial W_{k}^{\prime}$, i.e. the set of points of the form $n \exp k H \cdot o$ in $\partial W_{k}^{\prime}$, and define

$$
f_{k}(n)= \begin{cases}f(n \exp k H \cdot o) & \text { if } n \exp k H \cdot o \in S_{k}, \\ 0 & \text { otherwise. }\end{cases}
$$

Let $F_{k}$ be the Poisson integral of $f_{k}$ and $\Phi$ the Poisson integral of the characteristic function $\chi_{D^{\prime}}$ of the complement $D^{\prime}$ of $D$.

We claim that for some positive $A, B$ independent of $k$ we have, on $W_{k}^{\prime}$,

$$
F_{k}+A \Phi+B e^{-m t}>f-2 \epsilon .
$$

By harmonicity it suffices to prove this on $\partial W_{k}^{\prime}$. If $n \exp t H \cdot o \in S_{k}$ (the bottom), by our construction we have, for $n_{1} \in n b\left(R e^{-k}\right)$,

$$
\left(f_{k}+A \chi_{D^{\prime}}\right)\left(n_{1} \exp k H \cdot o\right)>f_{k}(n \exp k H \cdot o)-\epsilon
$$

(we only have to make sure that $A \geqslant|f|$ on $W_{\alpha}$ ). Thus Lemma 9 gives the desired inequality on $S_{k}$. On the "side", i.e. if $n \exp t H \cdot o \in \partial W_{k}^{\prime}(0<t<k)$, we have that $n \exp t H \notin \Gamma_{\alpha}\left(n^{\prime}\right)$ for all $n^{\prime} \in D$; thus $n b\left(\alpha e^{-t}\right) \subset D^{\prime}$. Now

$$
\Phi(n \exp t H \cdot o)=\left(\chi_{D^{\prime}} * P_{t}\right)(n) \geqslant \int_{n b\left(\alpha e^{-t}\right)} P_{t}\left(n_{1}\right) d n_{1}
$$

and by the homogeneity of $P_{t}$ the right-hand side is a positive constant independent of $t$. So, choosing $A$ sufficiently large, we have the claim on the "side". Finally, by choosing $B$ large enough we have the claim on the "top", and hence in all $W_{k}^{\prime}$.

Now applying the same argument to $-f$ in place of $f$, we have, on $W_{k}^{\prime}$,

$$
-F_{k}+A \Phi+B e^{-m t}>-f-2 \epsilon .
$$

Now we put these inequalities together, and let $k \rightarrow \infty .\left\{f_{k}\right\}$ has a weakly convergent subsequence tending to some function $f_{0}$; denoting the Poisson integral of $f_{0}$ by $F$, we have,

$$
F-A \Phi-B e^{-m t}-2 \epsilon<f<F+A \Phi+B e^{-m t}+2 \epsilon
$$


everywhere on $W_{\alpha}(D) . F$ is admissibly convergent almost everywhere, $\Phi$ and $e^{-m t}$ have admissible limit 0 almost everywhere in $D$. Hence, using Lemma 2, the oscillation of $f$ in any admissible domain at almost every $n \in D$ is less than $4 \epsilon$. Since $\epsilon$ and $\eta$ were arbitrary, the proof is complete.

\title{
REFERENCES
}

1. A. P. Calderón, On the behavior of harmonic functions at the boundary, Trans. Amer. Math. Soc. 68 (1950), 47-54. MR 11, 357.

2. S. Helgason, Differential geometry and symmetric spaces, Pure and Appl. Math., vol. 12, Academic Press, New York, 1962. MR 26 \#2986.

3. Duality and Radon transform for symmetric spaces, Amer. J. Math. 85 (1963), 667-692. MR 28 \#1632.

4. A. Korányi, Harmonic functions on Hermitian hyperbolic space, Trans. Amer. Math. Soc. 135 (1969), 507-516. MR 43 \#3480.

5. Boundary behavior of Poisson integrals on symmetric spaces, Trans. Amer. Math. Soc. 140 (1969), 393-409. MR 39 \#7132.

6. R. B. Putz, A generalized area theorem for harmonic functions on Hermitian hy. perbolic space, Trans. Amer. Math. Soc. 168 (1972), 243-258. MR 45 \#7101.

7. E. M. Stein, On the theory of harmonic functions of several variables. II. Behavior near the boundary, Acta Math. 106 (1961), 137-174. MR 30 \#3234.

8. - Boundary behavior of holomorphic functions of several complex variables, Princeton Univ. Press, Princeton, N. J., 1972.

9. A. Zygmund, Trigonometric series, Vols. I, II, 2nd rev. ed., Cambridge Univ. Press, New York, 1959. MR 21 \#6498.

\author{
BELFER GRADUATE SCHOOL OF SCIENCE, YESHIVA UNIVERSITY, NEW \\ YORK, NEW YORK 10033
}

